\title{
On a conjecture by San Pedro
}

\author{
Leszek Wroński
}

Received: 11 September 2013 / Accepted: 4 April 2014 / Published online: 7 June 2014 (C) The Author(s) 2014. This article is published with open access at Springerlink.com

\begin{abstract}
In a recent paper in this Journal Iñaki San Pedro put forward a conjecture regarding the relationship between no-conspiracy and parameter independence in EPR scenarios; namely, that violation of the former implies violation of the latter. He also offered an argument supporting the conjecture. In this short note I present a method of constructing counterexamples to the conjecture and point to a mistake in the argument.
\end{abstract}

Keywords EPR $\cdot$ Parameter independence $\cdot$ No conspiracy $\cdot$ Measurement independence

\section{Introduction}

Since the EPR correlations and the philosophical issues concerning them have been described by (San Pedro 2012), to which paper this note is a technical follow-up, I will be very brief here. In the setup with which we are concerned two electrons are emitted in opposite directions. Each electron from a given pair proceeds towards a detector which can be set to measure the electron's spin along an axis chosen from a previously determined set of directions (typically from 2 or 3 options); the results of the measurement are binary: either "spin-up" or "spin-down". Very roughly, the EPR correlations consist in the fact that while the probability of each result for any measurement direction in any of the two wings of the experiment is one-half, the probabilities of obtaining pairs of results (one from each wing) typically differ from one-fourth. This is puzzling due to the spatiotemporal features of the setup which

\footnotetext{
L. Wroński

Leszek Wroński Institute of Philosophy, Jagiellonian University, Grodzka 52, 31-044 Kraków, Poland e-mail: leszek.wronski@uj.edu.pl
} 
suggest various independencies: the outcome at one wing cannot influence the outcome in the other wing, and the choice of measurement setting at one wing cannot influence the outcome in the other wing.

The famous 1964 result of Bell shows that one cannot account for the correlations by positing a hidden variable subject to certain formal conditions which are supposed to formulate the natural independencies like the ones described above. There have been many attempts to formulate alternative explanations by means of various constructs subject to various independence relations; one usual relation of this sort is that of no conspiracy, or measure independence: the posited hidden variable should be statistically independent from the measurement settings. It is to this field that San Pedro makes a contribution. Apart from philosophical points about backwards causation, which will not concern me here, he offers a conjecture; namely, that whenever measurement independence is violated, the so called parameter independence condition-which says that if we fix the value of the posited hidden factor the chance for obtaining a given result in one wing should not depend on the measurement setting in the other wing - is also violated. San Pedro offers a mathematical argument supporting this conjecture. In the following sections I will present a method of constructing counterexamples to the conjecture and point to a flaw in San Pedro's argument.

\section{The formal setup}

San Pedro's notational conventions dictate that we use:

- $\quad L_{i}$ for the event of the detector in the left wing being set to the axis $i$;

- $\quad R_{j}$ for the event of the detector in the right wing being set to the axis $j$;

- $L_{i}^{a}$ for the event of the detector in the left wing, set to the axis $i$, displaying the result $a$;

- $\quad R_{j}^{b}$ for the event of the detector in the right wing, set to the axis $j$, displaying the result $b$;

where $i, j \in\{1,2,3\}$ and $a, b \in\{+,-\}$.

For the hidden causal factors San Pedro proposes to use the notation $C_{i j}^{a b}$, with $i, j, a, b$ as before. Now, due to the difference between "common common causes" and "separate common causes" (see e.g. Hofer-Szabó et al. 2013) it is currently standard to require different causal factors screening off correlations at different measurement settings. However, San Pedro aims to go one step further, and require that at the measurement setting $L_{i} \wedge R_{j}$, the event $C_{i j}^{a b}$ should screen off the correlation between $L_{i}^{a}$ and $R_{j}^{b}$ and none of the other three (anti-)correlations. That is, for example, $C_{13}^{++}$should screen off $L_{1}^{+}$from $R_{3}^{+}$, but not $L_{1}^{+}$from $R_{3}^{-1} \cdot$

This seems to be a mistake. Any event screening off one correlation at a given combination of measurement settings screens off all the other correlations at the same pair of measurement settings. Let me show an example of this (the argument is

\footnotetext{
${ }^{1}$ The occurrence of $C_{13}^{++}$does not in general entail the occurence of $L_{1}^{+} \wedge R_{3}^{+}$; i.e., we are not assuming the $C_{i j}^{a b}$ 's generally act as deterministic screening-off factors.
} 
similar in all other cases): if $C_{13}^{++}$screens off $L_{1}^{+}$from $R_{3}^{+}$, then it also screens off $L_{1}^{+}$from $R_{3}^{-}$.

Assume that (*) $P\left(L_{1}^{+} \wedge R_{3}^{+} \mid L_{1} \wedge R_{3} \wedge C_{13}^{++}\right)=P\left(L_{1}^{+} \mid L_{1} \wedge R_{3} \wedge C_{13}^{++}\right)$. $P\left(R_{3}^{+} \mid L_{1} \wedge R_{3} \wedge C_{13}^{++}\right)$. Now, $P\left(L_{1}^{+} \wedge R_{3}^{-} \mid L_{1} \wedge R_{3} \wedge C_{13}^{++}\right)=P\left(L_{1}^{+} \mid L_{1} \wedge R_{3} \wedge\right.$ $\left.C_{13}^{++}\right)-P\left(L_{1}^{+} \wedge R_{3}^{+} \mid L_{1} \wedge R_{3} \wedge C_{13}^{++}\right)$. Due to (*), this is equal to $P\left(L_{1}^{+} \mid L_{1} \wedge R_{3} \wedge\right.$ $\left.C_{13}^{++}\right)-P\left(L_{1}^{+} \mid L_{1} \wedge R_{3} \wedge C_{13}^{++}\right) \cdot P\left(R_{3}^{+} \mid L_{1} \wedge R_{3} \wedge C_{13}^{++}\right)$, which in turn equals $P\left(L_{1}^{+} \mid L_{1} \wedge R_{3} \wedge C_{13}^{++}\right) \cdot\left(1-P\left(R_{3}^{+} \mid L_{1} \wedge R_{3} \wedge C_{13}^{++}\right)\right)$. The last expression is just $P\left(L_{1}^{+} \mid L_{1} \wedge R_{3} \wedge C_{13}^{++}\right) \cdot P\left(R_{3}^{-} \mid L_{1} \wedge R_{3} \wedge C_{13}^{++}\right)$. Putting all this together, we get the result that $P\left(L_{1}^{+} \wedge R_{3}^{-} \mid L_{1} \wedge R_{3} \wedge C_{13}^{++}\right)=P\left(L_{1}^{+} \mid L_{1} \wedge R_{3} \wedge\right.$ $\left.C_{13}^{++}\right) \cdot P\left(R_{3}^{-} \mid L_{1} \wedge R_{3} \wedge C_{13}^{++}\right)$. And so the screening off we wanted to establish holds.

San Pedro's notation seems to be redundant, then. There is no great harm in keeping it, though; we will simply consider the existence of various screening-off factors for the correlations at a given pair of measurement settings.

We can now formulate the well-known independence conditions. The screening off condition is also called outcome independence (OI, p. 147):

$$
P\left(L_{i}^{a} \wedge R_{j}^{b} \mid L_{i} \wedge R_{j} \wedge C_{i j}^{a b}\right)=P\left(L_{i}^{a} \mid L_{i} \wedge R_{j} \wedge C_{i j}^{a b}\right) \cdot P\left(R_{j}^{b} \mid L_{i} \wedge R_{j} \wedge C_{i j}^{a b}\right)
$$

The other two requirements are parameter independence (PI, p. 153) and measurement independence (MI, p. 147; also called no conspiracy), each of which consists of a symmetric pair of conditions:

$$
\begin{gathered}
P\left(L_{i}^{a} \mid L_{i} \wedge C_{i j}^{a b}\right)=P\left(L_{i}^{a} \mid L_{i} \wedge R_{j} \wedge C_{i j}^{a b}\right) \\
P\left(R_{j}^{b} \mid R_{j} \wedge C_{i j}^{a b}\right)=P\left(R_{j}^{b} \mid L_{i} \wedge R_{j} \wedge C_{i j}^{a b}\right) \\
P\left(C_{i j}^{a b} \wedge L_{i}\right)=P\left(C_{i j}^{a b}\right) \cdot P\left(L_{i}\right) \\
P\left(C_{i j}^{a b} \wedge R_{j}\right)=P\left(C_{i j}^{a b}\right) \cdot P\left(R_{j}\right)
\end{gathered}
$$

Notice that the notion of measurement independence used by San Pedro is relatively weak: it does not require the independence of $C_{i j}^{a b}$ 's and pairs of measurement settings, and neither the independence of measurement settings and Boolean combinations of $C_{i j}^{a b}$, s (which are non-trivial for different pairs of measurement settings). Since I am commenting on a conjecture by San Pedro, I will keep his nomenclature.

Apart from the disjointness assumption, i.e. that with fixed $i$ and $j$, for $a \neq c$, $b \neq d, C_{i j}^{a b} \cap C_{i j}^{a d}=C_{i j}^{a b} \cap C_{i j}^{c b}=\emptyset$, on p. 146 San Pedro sets down another requirement for $C_{i j}^{a b}$, from which it follows that

$$
C_{i j}^{a b} \subset L_{i} \cap R_{j}
$$

which I will call the subset condition. ${ }^{2}$

I will not discuss the philosophical advantages or disadvantages of the subset condition, but will notice that, by mere mathematics, it renders San Pedro's conjecture

\footnotetext{
${ }^{2}$ Following San Pedro, when writing about probability of the conjunction of two events I will use the " $\wedge$ " sign, but when the context is clearly set-theoretical I will use " $\cap$ " instead.
} 
less interesting that it (I think) would be if the condition was removed (I consider this option in Sections 3.1 and 3.2).

First, San Pedro himself notices (p. 147) that his $C_{i j}^{a b}$ 's violate MI. Note that it is due to the subset condition and nothing else. It is elementary that if $A \subset B$ and $P(B) \neq 1$, then $P(A) \cdot P(B) \neq P(A \wedge B)$. The subset condition gives us $C_{i j}^{a b} \subset L_{i}$, and since there is more than one possible measurement setting in the left wing of the experiment, $P\left(L_{i}\right) \neq 1$. Therefore $P\left(C_{i j}^{a b} \wedge L_{i}\right) \neq P\left(C_{i j}^{a b}\right) \cdot P\left(L_{i}\right)$, that is, MI is violated.

Second, notice that due to the subset condition (and, again, nothing else) PI universally holds. This is because it follows immediately from the subset condition that $L_{i} \cap C_{i j}^{a b}=L_{i} \cap R_{j} \cap C_{i j}^{a b}$ (as both sides are identical to $C_{i j}^{a b}$ ). Therefore $P\left(L_{i}^{a} \mid L_{i} \wedge C_{i j}^{a b}\right)=P\left(L_{i}^{a} \mid L_{i} \wedge R_{j} \wedge C_{i j}^{a b}\right)$; similarly for the symmetric condition. Therefore PI always holds.

\section{The conjecture and the argument}

What is the conjecture, then? To quote San Pedro (p. 152):

Conjecture 1 If measurement independence is violated then parameter independence is also violated.

By the light of the previous section, we have to say that the conjecture is simply false. If we use San Pedro's definition, we cannot even contemplate a violation of PI.

At this point one may be tempted to consider an alternative formulation of parameter independence; namely, for $j \neq k$ and $h \neq i$ :

$$
\begin{aligned}
& P\left(L_{i}^{a} \mid L_{i} \wedge R_{k} \wedge C_{i j}^{a b}\right)=P\left(L_{i}^{a} \mid L_{i} \wedge R_{j} \wedge C_{i j}^{a b}\right) \\
& P\left(R_{j}^{b} \mid L_{h} \wedge R_{j} \wedge C_{i j}^{a b}\right)=P\left(R_{j}^{b} \mid L_{i} \wedge R_{j} \wedge C_{i j}^{a b}\right)
\end{aligned}
$$

Notice, though, that due to the subset condition (and the fact that $j \neq k$ and $h \neq i) L_{i} \cap R_{k} \cap C_{i j}^{a b}=L_{h} \cap R_{j} \cap C_{i j}^{a b}=\emptyset$, so $P\left(L_{i} \wedge R_{k} \wedge C_{i j}^{a b}\right)=$ $P\left(L_{h} \wedge R_{j} \wedge C_{i j}^{a b}\right)=0$, and thus the left-hand side probabilities in the PI' conditions are undefined. So PI' universally fails. On this formulation of parameter independence, the conjecture is true, but not interesting, akin to an implication with a previously known consequent. And again, the subset condition is the culprit. (From now on I will stick to PI as opposed to PI', since it is the formulation used by San Pedro.)

I suspect San Pedro himself had second thoughts about the subset condition, since he offered an argument in support of the conjecture, the falsity of which we have seen to follow directly from the subset condition. It might be interesting, then, to consider the conjecture without assuming SC. 
3.1 Without the subset condition: flaw in the argument

Before we tackle the conjecture itself, let us look at the argument for it given by San Pedro on p. 153. Consider the following excerpt:

A violation of measurement independence entails that (...)

$$
\begin{gathered}
P\left(C_{i j}^{a b} \wedge L_{i}\right) \neq P\left(C_{i j}^{a b}\right) \cdot P\left(L_{i}\right), \\
P\left(C_{i j}^{a b} \wedge R_{j}\right) \neq P\left(C_{i j}^{a b}\right) \cdot P\left(R_{j}\right),
\end{gathered}
$$

which entails that

$$
P\left(C_{i j}^{a b} \wedge L_{i} \wedge R_{j}\right) \neq P\left(C_{i j}^{a b}\right) \cdot P\left(L_{i} \wedge R_{j}\right),
$$

as long as we assume that $L_{i}$ and $R_{j}$ are probabilistically independent.

Notice again that in the presence of SC (20) would need no argument. If we forget about SC, though, the entailment does not hold: it is possible to make (18) and (19) true while making (20) false, while keeping $L_{i}$ and $R_{j}$ probabilistically independent. Consider the probability space with $\Omega=[0,1] \times[0,1]$, the event algebra $\mathcal{F}$ being the smallest $\sigma$-algebra containing all "rectangles" of the form "a Borel subset of $[0,1]$ $\times$ a Borel subset of $[0,1]$ ", and the measure being the unique extension to the whole $\mathcal{F}$ of the function $\operatorname{Pr}$ defined on the rectangles so that $\operatorname{Pr}(A \times B)=L(A) \cdot L(B)$, where $L$ is the Lebesgue measure. (In other words, consider a space defined over a square of area 1 , with the probability of its measurable subsets being their area.) Now define the following: ${ }^{3}$

$$
\begin{aligned}
L_{1}= & {[0,1] \times[1 / 2,1] } \\
L_{2}= & \Omega \backslash L_{1} ; \\
R_{1}= & {[0,1 / 2] \times[0,1] ; } \\
R_{2}= & \Omega \backslash R_{3} ; \\
C_{12}^{++}= & {[0,1 / 2] \times[1 / 4,1 / 2] \cup[0,1 / 2] \times[7 / 8,1] \cup } \\
& \cup(1 / 2,1] \times[1 / 8,1 / 2] \cup(1 / 2,1] \times[3 / 8,1] .
\end{aligned}
$$

The Reader may check that in this situation, after substituting $L_{1}$ for $L_{i}, R_{2}$ for $R_{j}$, and $C_{12}^{++}$for $C_{i j}^{a b}$, inequalities (18) and (19) hold, " $L_{i}$ and $R_{j}$ are probabilistically independent", but inequality (20) is false.

Of course, San Pedro only intended the argument to inspire more confidence in the truth of the conjecture, without giving an exact proof (he would not call it a "conjecture" in that case!); nonetheless, I think it is fitting when arguing against a conjecture supported by an argument to also point to a flaw in the argument. I can now turn to presenting the method of constructing counterexamples to San Pedro's conjecture.

\footnotetext{
${ }^{3}$ For brevity I am defining the space for two possible measurement settings at each detector (San Pedro considers three) but it should be obvious how to extend the example to cover more settings.
} 
3.2 Without the subset condition: falsity of the conjecture

In this section I will show how, given a probability space modelling some set of EPR correlations, that is, containing the events $L_{i}, R_{j}, L_{i}^{a}$ and $R_{j}^{b}$, to add to it events $C_{i j}^{a b}$ so that MI is violated while PI is satisfied. The construction will also satisfy OI. For an easier argument (saving us from an additional argument by cases), assume the (anti-)correlations are not perfect, i.e. that any combination of $L_{i}^{a} \wedge R_{j}^{b}$ occurs with a non-zero probability.

We can without loss of generality assume the probability space is atomless; that is, given an event $A$ with $P(A)>0$, we can always find an event $B$ such that $B \subseteq A$ and $P(A)>P(B)>0$. We do not lose any generality because any classical probability space can be embedded in an atomless classical probability space (see chapter 4 of Wroński 2010 for a short proof).

I will also again assume that we have two possible settings at each of the detectors. This is just for brevity, the reasoning goes through for any finite number of settings. I will label the directions for the left wing " 1 " and "2", and the other two " 3 " and " 4 ". Therefore the events of selecting the measurement directions are $L_{1}, L_{2}, R_{3}$ and $R_{4}$. We can think of the sample space as divided into four "quadrants" of the form $L_{i} \cap R_{j}$. So to define each $C_{i j}^{a b}$ we need to set its intersection with each quadrant, making sure the resulting event satisfies $\mathrm{OI}$ and PI. For example, for any $a, b \in\{+,-\}, C_{13}^{a b}$ should screen off all the (anti-)correlations between $L_{1}^{c}$ and $R_{3}^{d}$ (for any $c, d \in\{+,-\}$ ); also, the chance of $L_{1}^{c}$ should be the same conditional on $L_{1} \wedge C_{13}^{a b}$ as conditional on $L_{1} \wedge R_{3} \wedge C_{13}^{a b}$, and so on.

The key observation is the following: for any $C_{i j}^{a b}$, making sure it satisfies $\mathrm{OI}$ and PI only requires paying attention to its intersection with three quadrants. For example, in the case of $C_{13}^{++}$, we need to specify its intersection with $L_{1} \cap R_{3}$ (for OI to work) and with $L_{1} \cap R_{4}$ and $L_{2} \cap R_{3}$ (for PI to work). Its intersection with the last remaining quadrant, $L_{2} \cap R_{4}$, is irrelevant for the purposes of establishing OI and PI. Once the intersections of $C_{13}^{++}$with three quadrants are fixed, MI might already be violated. But if it is not, then there is at most one number fit for the probability of $P\left(C_{13}^{++} \wedge L_{2} \wedge R_{4}\right)$ if MI is to be sustained; label the number $r$. But since we are (due to atomlessness) at our complete liberty when it comes to choosing the probability of $P\left(C_{13}^{++} \wedge L_{2} \wedge R_{4}\right)$, we can choose the event so that its probability does not equal $r$, therefore violating MI. (In all but some very exceptional cases, ${ }^{4}$ setting the intersection $C_{13}^{++} \cap L_{2} \cap R_{4}$ to be the empty set will be enough to the trick.) And so what is really needed is only the way of defining $C_{13}^{++}$'s intersection with the three abovementioned quadrants so that OI and PI are satisfied.

This we will achieve in the following way. For each pair of detector settings $i, j$ we will consider four events: $C_{i j}^{++}, C_{i j}^{-+}, C_{i j}^{+-}$, and $C_{i j}^{--}$. The intuitive meaning is

\footnotetext{
${ }^{4}$ Exactly: if the numerator of the fraction at the right-hand side of the identity (**) below is not equal to 0 .
} 
that $C_{i j}^{a b}$ should guarantee ${ }^{5}$, if we set the left detector to $i$ and the right detector to $j$, the occurence of $L_{i}^{a} \wedge R_{j}^{b}$. In the particular case of $C_{13}^{++}$we will achieve this (which means that $\mathrm{OI}$ is satisfied), as well as the satisfaction of PI, by setting the intersection of $C_{13}^{++}$with the three relevant quadrants in the following way:

$$
\begin{aligned}
C_{13}^{++} \cap L_{1} & :=\left(L_{1}^{+} \cap R_{3}^{+}\right) \cup\left(L_{1}^{+} \cap R_{4}^{+}\right), \\
C_{13}^{++} \cap L_{2} \cap R_{3} & :=L_{2}^{+} \cap R_{3}^{+} .
\end{aligned}
$$

With $C_{13}^{+-}$we set the intersections as follows:

$$
\begin{aligned}
C_{13}^{+-} \cap L_{1} & :=\left(L_{1}^{+} \cap R_{3}^{-}\right) \cup\left(L_{1}^{+} \cap R_{4}^{-}\right), \\
C_{13}^{+-} \cap L_{2} \cap R_{3} & :=L_{2}^{+} \cap R_{3}^{-},
\end{aligned}
$$

with the remaining two cases dealt with analogously. It is elementary that the $C_{13}^{a b}$ 's defined in this way satisfy OI and PI no matter what their intersection with $L_{2} \cap R_{4}$ is.

We now need to ensure the violation of MI. Notice that MI requires that

$$
P\left(C_{13}^{++}\right) \cdot P\left(L_{2}\right)=P\left(C_{13}^{++} \wedge L_{2} \wedge R_{3}\right)+P\left(C_{13}^{++} \wedge L_{2} \wedge R_{4}\right),
$$

which we can transform into

$$
P\left(C_{13}^{++} \wedge L_{2} \wedge R_{4}\right)=\frac{\left(P\left(C_{13}^{++} \wedge R_{3}\right)+P\left(C_{13}^{++} \wedge L_{1} \wedge R_{4}\right)\right) \cdot P\left(L_{2}\right)-P\left(C_{13}^{++} \wedge L_{2} \wedge R_{3}\right)}{P\left(L_{1}\right)}
$$

Notice that the values of all the terms on the right hand side of the equality are known. Label the value of the fraction $r$. We now know that if we set the intersection $C_{13}^{++} \cap L_{2} \cap R_{4}$ so that its probability is different than $r$, MI will be violated. Thanks to atomlessness, there are infinitely many ways of doing so. ${ }^{6}$

At this point we have secured the violation of MI. As I have already stated, all $C_{13}^{a b}$ 's satisfy OI and PI. Now all that needs to be done is to repeat the above construction for $C_{14}^{a b}$, s, $C_{23}^{a b}$, and $C_{24}^{a b}$, changing the role of the quadrants accordingly.

Notice also that, for any $i$ and $j$, nothing prevents us from setting the events in the "irrelevant" quadrant so that the $C_{i j}^{a b}$ 's form a partition of the sample space.

We now know that if we remove the subset condition, the conjecture is false. I have presented the construction for imperfect (anti-)correlations, but it should be evident how to transform it into one fit for that special case.

\footnotetext{
${ }^{5}$ Please notice that this interpretaion of $C_{i j}^{a b}$ is intended only in my construction and not in San Pedro's approach (though it of course permits it); in my counterexamples the $C_{i j}^{a b}$ act as "deterministic" screeningoff factors. San Pedro does not require this in his framework. It is just that I think using screening-off factors which are deterministic is the simplest way to find counterexamples.

${ }^{6}$ If we do not assume atomlessness, we can just set the intersection to be the empty set or, in the unlikely case in which $r=0$, to be the full quadrant $L_{2} \cap R_{4}$; then we would set the intersection of $C_{13}^{+-}, C_{13}^{-+}$, and $C_{13}^{--}$with $L_{2} \cap R_{4}$ to be empty.
} 


\section{Conclusions}

In the previous section I argued that San Pedro's conjecture, taken as a mathematical statement (since its proponent himself gives a mathematical argument in its support) is false. I showed that if we keep San Pedro's definitions, the conjecture fails immediately; while if we remove one of the conditions, it is still possible to provide a method for obtaining (infinitely many) counterexamples. Now, the counterexamples are mathematical constructions. It would be interesting if there were philosophical reasons for rejecting them, especially if some of the reasons could arguably result in additional formal requirements which my construction fails to satisfy. I would welcome such a development; as it stands, though, San Pedro's conjecture is false.

Acknowledgments I would like to thank Juliusz Doboszewski, Michał Marczyk and Tomasz Placek for commenting on the initial version of the manuscript and also the two anonymous reviewers for their helpful comments. My research has been aided by the Foundation for Polish Science "START" Fellowship and also by the Foundation for Polish Science "MISTRZ" Fellowship received by Tomasz Placek.

Open Access This article is distributed under the terms of the Creative Commons Attribution License which permits any use, distribution, and reproduction in any medium, provided the original author(s) and the source are credited.

\section{References}

Hofer-Szabó, G., Rédei, M., Szabó, L.E. (2013). The common cause principle. Cambridge University Press.

San Pedro, I. (2012). Causation, measurement relevance and no-conspiracy in EPR. European Journal for Philosophy of Science, 2, 137-156.

Wroński, L. (2010). The common cause principle. Explanation via screening off. $\mathrm{PhD}$ thesis, Jagiellonian University, Kraków. Archived at http://jagiellonian.academia.edu/LeszekWroński. Forthcoming as a book from De Gruyter Open. 